\title{
La surveillance de la qualité de l'air dans les Hauts-de-France
}

\author{
Jimmy Leyes, Laure Roussel \\ Atmo Hauts-de-France, Lille \\ I.roussel@atmo-hdf.fr
}

\section{Résumé}

La surveillance réglementaire de la qualité de l'air en France est confiée aux associations régionales agréées de surveillance de la qualité de l'air (Aasqa) telles qu'Atmo Hauts-deFrance. Elles s'appuient sur une palette d'outils et leur expertise pour mesurer les polluants dans l'air de leur territoire, alerter les populations en cas d'épisode de pollution, répondre aux exigences réglementaires de surveillance définies au niveau européen, tout en prenant en compte les spécificités régionales. Cet article présente les différents outils utilisés par les Aasqa, et plus particulièrement Atmo Hauts-deFrance, pour surveiller et estimer la qualité de l'air. L'association régionale opère ainsi un ensemble de stations de mesures fixes et mobiles pour suivre en continu les concentrations de polluants réglementés ou non sur son territoire, et dispose d'outils de modélisation pour évaluer et prévoir la qualité de l'air en tous points de la région. Cet article présente également certains des paramètres météorologiques qui influencent la qualité de l'air de la région Hauts-de-France, particulièrement concernée par les épisodes de pollution aux particules.
Londres en décembre 1952 (Davies, 2002). Au début des années 1970, plusieurs associations locales développent leurs propres réseaux de surveillance de la qualité de l'air. Aujourd'hui, elles sont devenues les associations régionales agréées de surveillance de la qualité de l'air (Aasqa). Au nombre de 19, elles sont approuvées par le ministère en charge de l'écologie et regroupées au sein de la fédération Atmo France (figure 1). Elles ont pour missions de surveiller et prévoir la qualité de l'air, informer et sensibiliser la population, accompagner les décideurs et améliorer les connaissances sur la pollution atmosphérique. Les techniques de mesures et les outils d'évaluation de la qualité de l'air permettent aujourd'hui de prévoir les pics de pollution, afin d'informer et d'avertir la population en cas de risque. Pour cela, les Aasqa s'appuient sur leur expertise, les données disponibles du territoire ainsi que sur les prévisions météorologiques. En effet, la qualité de l'air est fortement influencée par deux catégories de paramètres : la production de polluants par diverses sources de pollution (émissions), ainsi que les facteurs météorologiques et topographiques.

-2 août 1961 : Loi relative à la lutte contre les pollutions atmosphériques et les odeurs. Elle impose aux immeubles, aux établissements industriels, commerciaux, artisanaux ou agricoles et aux véhicules d'" être construits, exploités ou utilisés de manière à [...] éviter les pollutions de l'atmosphère et les odeurs qui incommodent la population, compromettent la santé ou la sécurité publique, ou nuisent à la production agricole, à la conservation des constructions et monuments ou au caractère des sites".

- Années 1970 : premiers réseaux de surveillance de la qualité de l'air.

- 30 décembre 1996 : Loi Laure (loi sur l'air et l'utilisation rationnelle de l'énergie). Elle reconnaît à chaque individu le " droit de respirer un air qui ne nuise pas à sa santé " et rend obligatoire sa surveillance au niveau local.

\section{Les polluants atmosphériques}

L'article R. 221-1 du code de l'environnement définit comme polluant atmosphérique " toute substance présente dans l'air ambiant et pouvant avoir des effets nocifs sur la santé humaine ou sur l'environnement dans son ensemble ». Il existe des dizaines de polluants présents dans l'atmosphère sous forme gazeuse ou sous forme de particules solides ou liquides en suspension. 


\section{Abstract}

\section{Air quality monitoring in the Hauts-de-France}

Regulatory air quality monitoring in France is performed by government-approved non-profit organisations called AASQAs, one of which is Atmo Hauts-de-France. These organisations rely on decades of accumulated air quality expertise and use several techniques to measure air pollutant concentrations, inform the public when pollutant levels are unhealthy, and comply with E.U. air quality monitoring regulations. This paper gives an overview of the tools used by AASQAs, and more particularly by Atmo Hauts-deFrance, to monitor and forecast air quality. The year-round continuous monitoring of air pollutant levels at fixed sites is supplemented by shortterm measurements made with fully-equipped vehicles or trailers and by modelling tools that forecast air quality and estimate pollutant levels where there are no measurements. AASQAs study pollutants which ambient concentrations are regulated by European air quality standards as well as other pollutants which are not regulated in this way. This work also discusses some of the meteorological factors, that affect air quality in the region Hauts-deFrance, which is heavily impacted by particulate matter pollution.

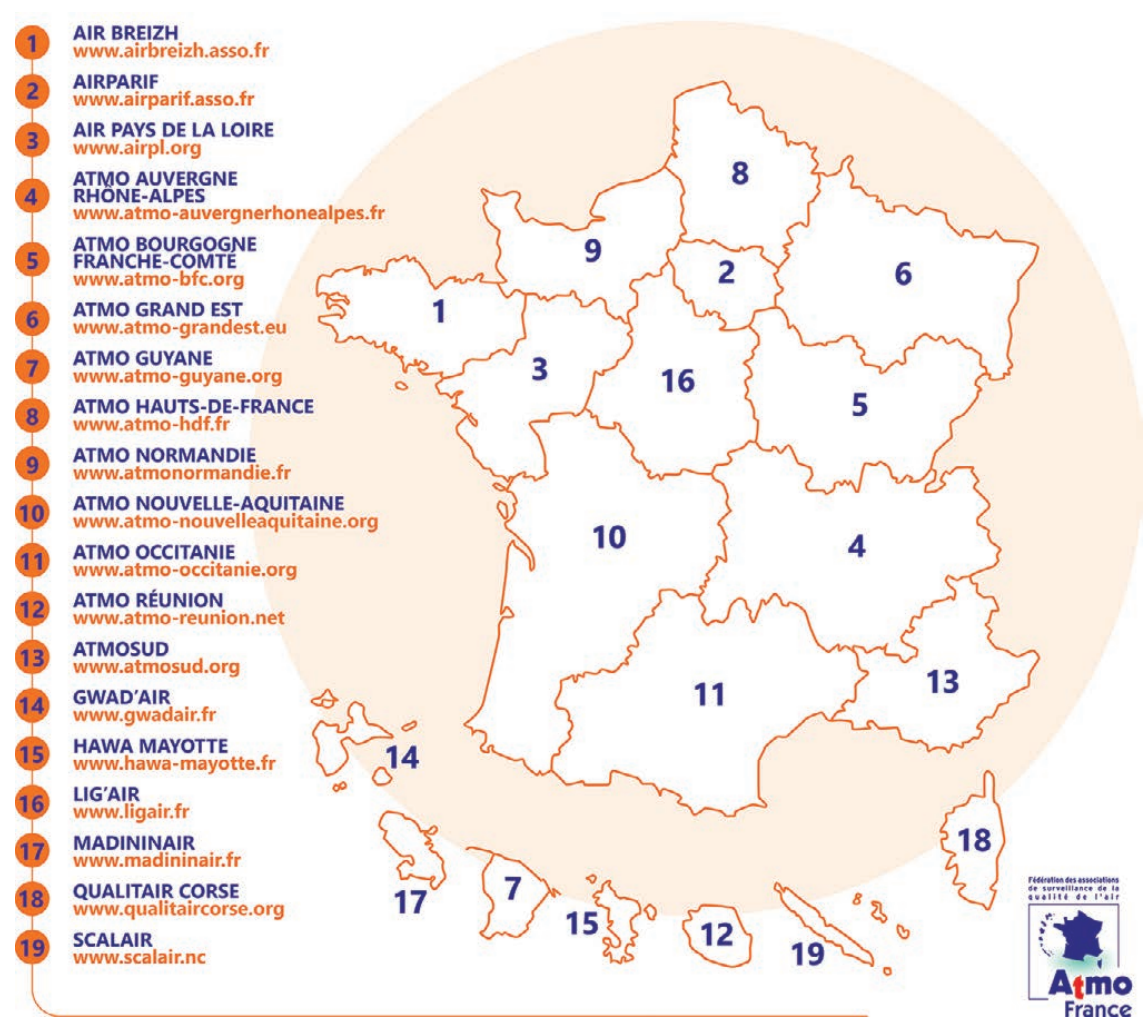

Figure 1. Carte des Aasqa. Source : Atmo France.

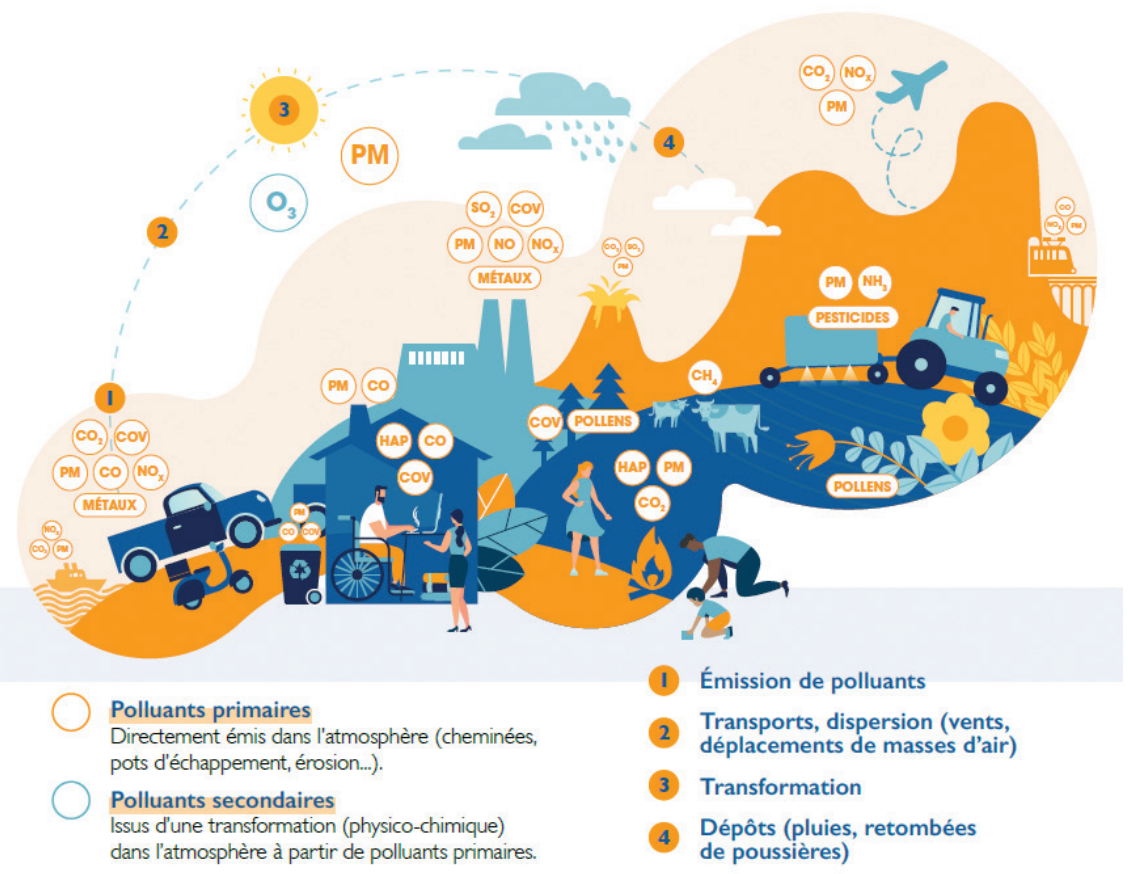

Figure 2. Représentation schématique des sources des principaux polluants de l'air surveillés par les Aasqa. Source : Atmo France.

Les Aasqa mesurent un large panel de polluants atmosphériques, réglementés ou non, intégrés dans les dispositifs de surveillance régionale. Douze polluants ou familles de polluants sont réglementés et surveillés (figure 2$)^{1}$ : l'ozone $\left(\mathrm{O}_{3}\right)$, le dioxyde d'azote $\left(\mathrm{NO}_{2}\right)$, le dioxyde de soufre $\left(\mathrm{SO}_{2}\right)$, les particules en suspension PM10 et PM2,5 (particules de diamètre inférieur ou égal à 10 et $2,5 \mu \mathrm{m})$, le monoxyde de carbone $(\mathrm{CO})$, les métaux lourds (cadmium, plomb, nickel, arsenic), le benzène (famille des composés organiques volatils ou COV) et le benzo[a] pyrène (famille des hydrocarbures

1. Atmo France, 2020. Un air sain pour tous. https://atmo-france.org/wp-content/uploads/ 2020/03/1790-AtmoFr-brochure_web.pdf, p. 6 . 
aromatiques polycycliques ou HAP). Des seuils réglementaires à ne pas dépasser pour chacun de ces polluants sont définis dans les directives européennes, déclinées en droit français par des décrets ou des arrêtés (cf. décret $\mathrm{n}^{\circ}$ 2010-1250 du 21 octobre 2010 relatif à la qualité de l'air). Chaque Aasqa réalise un bilan annuel à l'échelle de sa région pour constater les éventuels dépassements de ces valeurs réglementaires et pour évaluer l'exposition de la population et de la végétation à la pollution atmosphérique.

D'autres polluants, non réglementés, sont également surveillés par les Aasqa, pour répondre aux spécificités et aux enjeux de chaque territoire. Atmo Hauts-de-France surveille des polluants définis dans une stratégie nationale, comme les particules ultrafines, le carbone suie, etc. L'observatoire régional mesure aussi les pollens dans l'air depuis plus de 15 ans sur la commune de Lille (Desmettres, 2015). Il a également mené des études spécifiques sur le sujet dans les logements d'agriculteurs dans le Nord et le Pas-deCalais (Desmettres, 2013) et dans des lycées de Picardie (Escat, 2013), et a participé en 2018-2019 à la campagne exploratoire nationale de mesure de pesticides dans l'air ${ }^{2}$ (Quindroit, 2020). Des mesures de radioactivité ambiante sont également réalisées quotidiennement sur la Métropole européenne de Lille et la Communauté urbaine de Dunkerque en lien avec les acteurs locaux et régionaux, notamment pour répondre aux attentes locales et pour informer les populations $^{3}$. Pour répondre aux préoccupations de la population amiénoise et à la demande d'Amiens Métropole, Atmo Hauts-de-France a mis en place depuis 2007 un réseau de veille olfactive sur l'agglomération. Le réseau de « Nez » est composé de personnes bénévoles formées par Atmo à la reconnaissance des odeurs. Pour compléter ce réseau, une plateforme de signalements disponible sur l'application ODO, financée par l'agglomération amiénoise, a été créée par Atmo et permet de déclarer des nuisances olfactives.

Les polluants atmosphériques sont soit émis directement dans l'atmosphère (ce sont des polluants primaires), soit formés dans l'atmosphère à partir d'autres espèces, par des réactions chimiques ou physiques (ces polluants sont dits secondaires). Les sources d'émissions des polluants primaires sont issues d'activités humaines (transports, chauffage, agriculture, activités industrielles) ou d'origine naturelle (végétation, éruptions volcaniques, étendues d'eau, foudre, etc.). La région Hauts-de-France est située au cœur de l'Europe, sous l'influence des populations provenant de la Belgique, des départements du Grand-Est, de l'Îlede-France, de la Normandie, mais aussi de l'Angleterre. Troisième région la plus peuplée de France, avec une très forte densité de population dans les départements du Nord et du Pas-de-Calais, elle est marquée par une urbanisation et un réseau de transport très développés, la présence de zones industrielles (notamment autour de Calais, Dunkerque et l'ancien bassin minier) et de grandes surfaces agricoles. Présentant peu de relief, mais une grande diversité de milieux naturels (marais, forêts, cours d'eau, dunes, parcs naturels), la région est également bordée par la Manche et la mer du Nord.

\section{Les outils de surveillance de la qualité de I'air}

Pour évaluer la qualité de l'air, les Aasqa s'appuient sur leur stratégie régionale de surveillance de l'air. Elle est définie dans un programme quinquennal décliné à partir du Plan national de surveillance de la qualité de l'air. Ce programme détermine les moyens les plus adaptés pour mesurer la qualité de l'air, les orientations pour informer efficacement la population régionale et les partenariats sur lesquels s'appuyer, ainsi que la stratégie d'accompagnement des acteurs de la qualité de l'air (collectivités, acteurs économiques, etc.). La stratégie de surveillance de la qualité de l'air repose sur deux outils complémentaires : la mesure de polluants atmosphériques et l'estimation des concentrations de polluants atmosphériques en tous points du territoire par la modélisation.

\section{Typologies des mesures}

Chaque mesure de polluant vise un objectif de surveillance particulier. On distingue les mesures de fond et les mesures sous influence. Les mesures de fond peuvent être urbaines, périurbaines ou rurales, sans l'influence d'une source particulière d'émissions de polluants. Ces mesures représentent l'exposition moyenne de la population de la zone située autour de la station de mesures. Les mesures de proximité ou sous influence prédominante permettent de quantifier l'impact sur la population d'une source d'émission identifiée, comme une activité industrielle ou le trafic automobile.

\section{Le réseau fixe de mesures}

En Hauts-de-France, l'Observatoire régional de l'air, Atmo Hauts-deFrance, dispose aujourd'hui de près de 50 stations de mesures. Ces stations sont fixes et leur implantation sur le territoire répond aux exigences réglementaires nationales ${ }^{4}$ fixées par le Laboratoire central de surveillance de la qualité de l'air (LCSQA).

Répartis dans ces stations, plus de 140 analyseurs de mesures en continu $(24 \mathrm{~h} / 24)$ permettent de mesurer les concentrations de différents polluants dans l'air (figure 3). Cette surveillance fournit des résultats en temps réel et permet de suivre l'évolution de la concentration d'un polluant ou d'une famille de polluants en un point précis tout au long de l'année (encadré 2). Ces analyseurs mesurent l'ozone, les oxydes d'azote, le dioxyde de soufre, les particules PM10 et PM2,5, le monoxyde de carbone, le benzène, la radioactivité, etc. présents dans l'air. L'ensemble des mesures est transmis en direct sur les serveurs de l'observatoire et est accessible via le site internet www.atmo-hdf.fr.

Les stations fixes d'Atmo Hauts-deFrance accueillent également une quinzaine de préleveurs pour des mesures différées en certains points du territoire. Ces appareils piègent les polluants de l'air sur des filtres. Ces polluants sont récupérés quelques jours plus tard puis analysés en laboratoire, ce qui permet de mesurer les hydrocarbures aromatiques polycycliques (HAP), les métaux lourds (nickel, plomb, arsenic, cadmium...) ou les pesticides, par exemple.

Certaines stations de mesures sont également équipées de mâts météorologiques, notamment pour recueillir la direction et la vitesse du vent, la température, l'humidité et la pression atmosphérique (figure 3).

\section{Les mesures par moyens mobiles}

En complément des stations fixes, Atmo Hauts-de-France dispose de stations dites mobiles, elles aussi équipées

\footnotetext{
2. Rapport AST de l'Anses relatif aux premières interprétations des résultats de la Campagne nationale exploratoire des pesticides (CNEP) dans l'air ambiant, octobre 2020.

3. Atmo HdF, Dispositif de surveillance de la radioactivité, septembre 2016.
} 

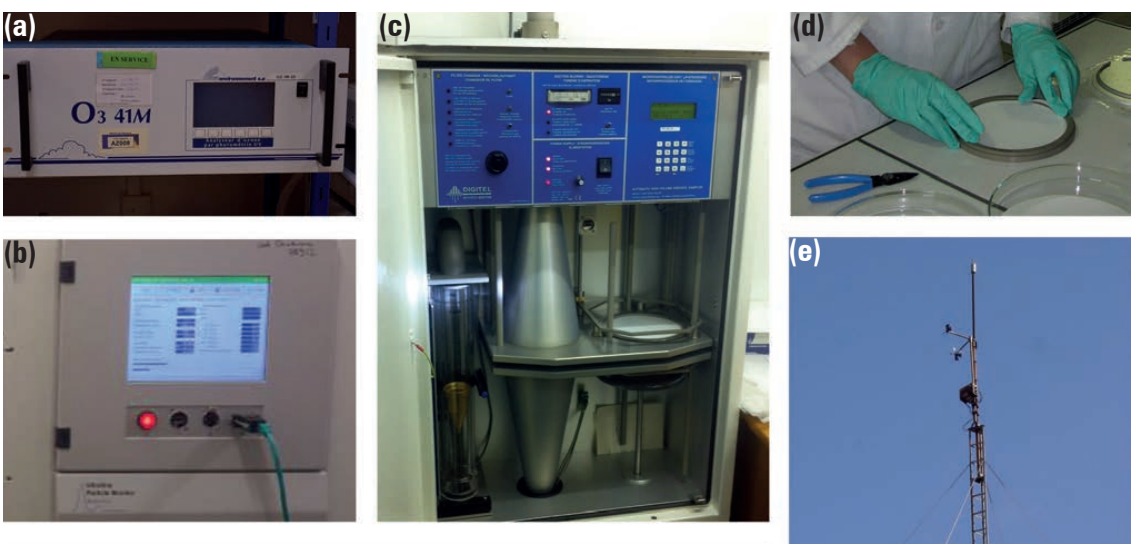

(f)
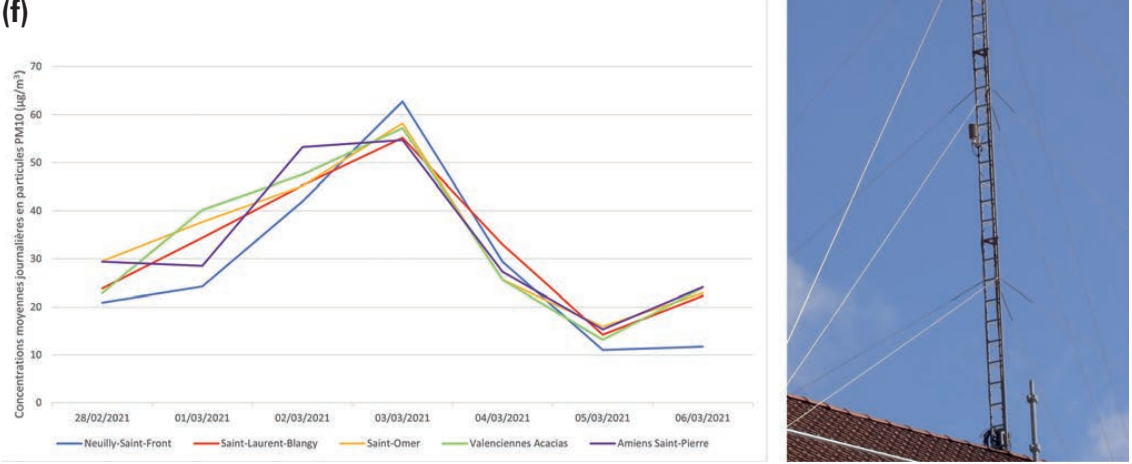

Figure 3. (a) Analyseur de gaz pour l'ozone. (b) Analyseur de particules ultrafines. (c) Préleveur. (d) Préparation de filtres en laboratoire. (e) Mât météorologique. (f) Séries temporelles des concentrations moyennes journalières en particules PM 10 du 28 février au 6 mars 2021 mesurées sur cinq stations de mesures. (c) Atmo Hauts-de-France.

de différents analyseurs et d'une station météo. Elles permettent la mesure de polluants de l'air sur des périodes définies (d'un à plusieurs mois) pour répondre à divers besoins ponctuels : surveillance de la qualité de l'air sur un territoire ne disposant pas de station fixe ou à proximité d'une source de pollution par exemple. À l'instar des analyseurs en stations fixes, l'ensemble des données est transmis en temps réel par le réseau.

Atmo Hauts-de-France utilise également des échantillonneurs passifs. $\mathrm{Ce}$ dispositif piège spécifiquement certains types de polluants sur un support contenu dans un tube exposé à l'air libre durant une période définie (de quelques heures à un mois selon les besoins). Les échantillons sont ensuite analysés en laboratoire pour déterminer la concentration moyenne d'un polluant durant la période d'exposition. Ce dispositif permet une mise en œuvre simple et rapide d'un dispositif de surveillance sur un grand nombre de sites simultanément, car ils sont peu encombrants et ne nécessitent pas d'alimentation électrique. Les échantillonneurs passifs sont principalement utilisés pour le dioxyde d'azote, le benzène ou l'ammoniac.

\section{L'évaluation et la prévision de la qualité de l'air grâce à la modélisation}

Les stations de mesure fixes et mobiles fournissent en temps réel ou en différé une information sur la qualité de l'air, mais leurs données sont représentatives sur quelques centaines de mètres au maximum, autour d'elles (la représentativité d'une station de fond est de l'ordre de quelques kilomètres carrés). Pour estimer et prévoir la qualité de l'air sur l'ensemble du territoire, Atmo Hautsde-France utilise des modèles de qualité de l'air. À l'instar des modèles de prévisions météorologiques, ceux-ci sont des simplifications/approximations des phénomènes réels. Il existe différents modèles utilisés en fonction notamment de la zone géographique à couvrir, des phénomènes que l'on souhaite représenter (grande échelle ou proximité), du délai attendu pour les résultats et de la puissance de calcul nécessaire pour faire fonctionner ces modèles.

Pour l'évaluation et la prévision de la qualité de l'air, Atmo Hauts-de-France utilise quotidiennement des modèles disponibles pour plusieurs polluants et à différentes échelles (figure 4) : la plateforme Prev'Air à l'échelle continentale et nationale $(5 \mathrm{~km}$ de résolution), la plateforme inter-régionale et régionale Esmeralda (3 $\mathrm{km}$ de résolution), pour répondre aux besoins réglementaires sur la prévision des épisodes de pollution et l'indice qualité de l'air. Ces modèles représentent la pollution de fond, représentative d'une large zone éloignée des sources de pollution (telles que le trafic ou les industries).

Ces plateformes sont une association d'un modèle météorologique, d'un modèle de chimie transport incluant des prétraitements, et un ensemble de post-traitements statistiques. Elles permettent de produire les cartes de concentrations en moyenne journalière ou en maximum journalier pour les particules PM10 et PM2,5, le dioxyde d'azote, l'ozone ou le dioxyde de soufre, au niveau du sol.

La plateforme fine échelle Urban'Air pour certaines agglomérations (jusqu'à $10 \mathrm{~m}$ de résolution) et maintenant la modélisation fine échelle régionale $(25 \mathrm{~m})$ permettent d'intégrer les phénomènes de pollution de proximité comme le trafic et d'évaluer l'exposition de la population au plus près. Les plateformes Urban'Air fonctionnent avec le modèle de dispersion ADMS Urban; elles permettent d'accéder à l'indice Atmo et aux sous-indices de qualité de l'air pour le dioxyde d'azote, l'ozone, les particules PM10, voire pour le dioxyde de soufre. Le modèle régional fine échelle est un modèle purement statistique, qui permet à ce jour de produire des cartes de concentrations en moyennes journalières pour les particules PM10 et PM2,5, et en maximum horaire pour le dioxyde d'azote et l'ozone.

La modélisation de la pollution atmosphérique se base sur un ensemble de paramètres qui sont intégrés et traités dans chaque type de modèle :

- les émissions de polluants atmosphériques d'origine naturelle ou liées aux activités humaines (transports, résidentiel/tertiaire, activités économiques...). Ces données proviennent des inventaires des émissions de polluants des Aasqa, qui recensent les quantités de chaque polluant émis par activité en tous points du territoire et pour une année de référence. L'inventaire d'Atmo Hauts-de-France intègre près de 50 polluants ou familles de polluants, dont six gaz à effet de serre ;

- les conditions aux limites et les conditions initiales d'un modèle à plus large échelle (pour les modèles Esmeralda et

4. Annexe 1.1 de l'arrêté du 17 juillet 2019 relatif au dispositif national de surveillance de la qualité de l'air ambiant. 

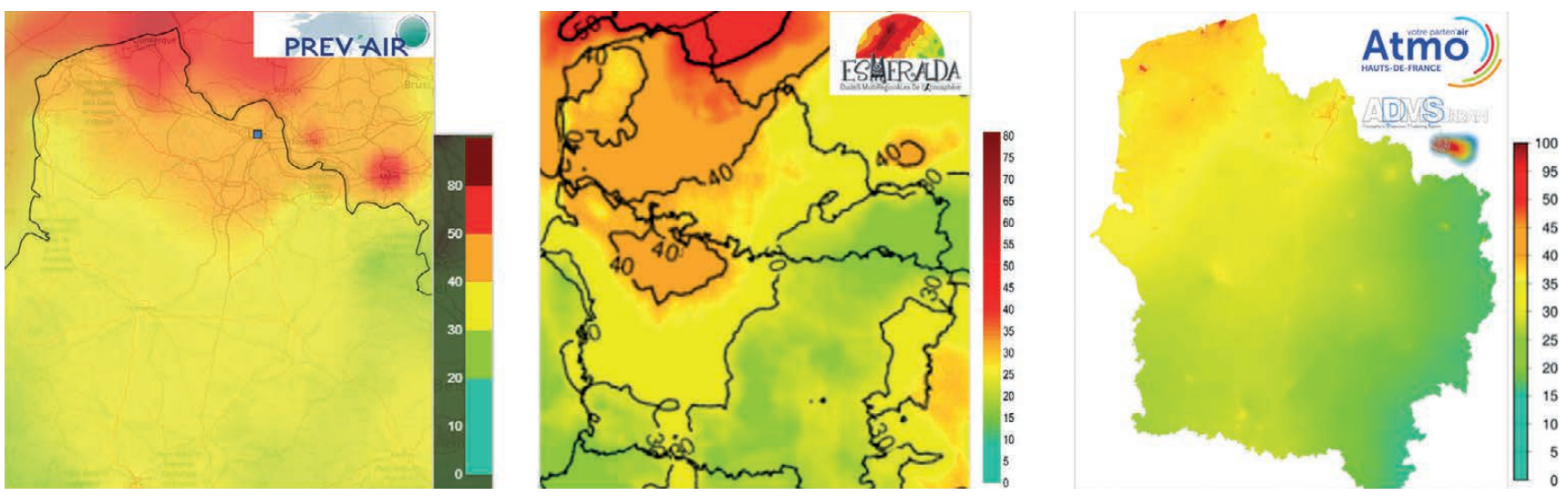

Figure 4. Prévisions de la qualité de l'air : Prev'Air (à gauche), Esmeralda (au centre) et prévision fine échelle régionale (à droite), pour les particules PM10 (concentrations en $\mu \mathrm{g} / \mathrm{m}^{3}$ ) en Hauts-de-France, le 31 mars 2021.

Prev'air), ou la pollution de fond provenant des données de mesures des stations du réseau d'Atmo Hauts-deFrance ou du modèle Prev'Air ;

- les prévisions et données météorologiques qui ont une influence sur la transformation chimique des polluants dans l'air et sur leur dispersion (développé dans la dernière partie de l'article);

- la topographie et l'occupation des sols, qui agissent aussi sur la dispersion et la turbulence des masses d'air.

Les Aasqa utilisent ces modèles de prévision de la qualité de l'air pour calculer les indices quotidiens de la qualité de l'air, ainsi que pour prévoir les épisodes de pollution.

\section{Les épisodes de pollution}

Chaque jour, les prévisionnistes des Aasqa évaluent le risque d'un épisode de pollution atmosphérique pour le jour-même et/ou le lendemain à partir des modèles de prévision de la qualité de l'air, des données météorologiques, des paramètres mesurés aux stations et de leur expertise. Un épisode de pollution atmosphérique correspond à une période où les concentrations de polluants dans l'air ne respectent pas ou risquent de ne pas respecter les seuils, selon des critères prédéfinis dans l'arrêté du 7 avril 2016 relatif au déclenchement des procédures préfectorales en cas d'épisodes de pollution de l'air ambiant (pourcentage de surface de la zone ou pourcentage de population impacté, seuil franchi, durée de l'épisode...). Le dispositif d'information et d'alerte concerne quatre polluants : l'ozone $\left(\mathrm{O}_{3}\right)$, le dioxyde d'azote $\left(\mathrm{NO}_{2}\right)$, le dioxyde de soufre $\left(\mathrm{SO}_{2}\right)$ et les particules en suspension PM10. Lorsqu'un épisode de pollution est prévu par les équipes d'Atmo Hauts-de-France pour le jour-même et/ou le lendemain, l'observatoire diffuse un communiqué chaque jour de l'épisode avant $12 \mathrm{~h}$ aux autorités compétentes ainsi qu'aux médias et à leurs abonnés.

L'information sur la base des prévisions permet aux autorités préfectorales d'anticiper les pics de pollution et aux populations, notamment les personnes les plus sensibles, d'adapter leurs activités en conséquence. Au niveau local, des arrêtés préfectoraux régissent les pratiques lors d'épisodes de pollution. L'arrêté interdépartemental d'information et d'alerte du public pour les Hauts-de-France du 5 juillet 2017 vise à protéger les populations lors des épisodes de pollution et à limiter la production de polluants. Le plan d'actions est gradué en fonction du seuil risquant d'être atteint, de l'étendue et la durée $\mathrm{du}$ phénomène de pollution. Il peut s'agir de la diffusion d'informations et de recommandations, de limitations de vitesse, de restrictions de la circulation automobile, de réductions des émissions liées à certaines activités industrielles ou agricoles, de la gratuité des transports en commun, etc.

Les Hauts-de-France sont régulièrement confrontés à des épisodes de pollution, notamment aux particules PM10 tout au long de l'année (encadré 3 et à l'ozone au printemps et en été. En 2019 et 2020, 51 et 28 journées de pollution, respectivement, ont été constatées sur la région, correspondant à 23 et 11 épisodes. Les départements du Nord et du Pas-de-Calais sont en général plus touchés par les épisodes que les autres départements de la région (Aisne, Oise et Somme). Ces épisodes, plus marqués sur certaines zones du territoire, peuvent s'expliquer en partie par des émissions importantes et localisées de particules et de polluants gazeux, pouvant former

\section{Les Hauts-de-France, une région concernée par les particules}

Les niveaux de PM10 et PM2,5 sont relativement élevés dans la région Hauts-deFrance, une problématique présente sur le territoire depuis plusieurs années. Étant un véritable enjeu sanitaire, ces particules sont doublement surveillées en région : par le réseau de stations fixes et par plusieurs outils de prévision permettant de modéliser leurs concentrations. De plus, chaque épisode de pollution aux particules PM10 fait l'objet d'une étude complète afin d'en identifier les origines. Une comparaison des données de mesures avec six autres Aasqa et leurs homologues belges montre que les moyennes des concentrations annuelles en 2019 pour les particules PM10 et PM2,5 des stations d'Atmo Hauts-de-France sont supérieures aux autres Aasqa. II en est de même pour les dépassements journaliers des seuils réglementaires (particules PM10) et recommandés (particules PM2,5). Selon Le Roux (2020) : "Ces valeurs de moyennes et dépassements relativement élevées par rapport aux autres Aasqa indiqueraient la présence d'émissions plus importantes (particules primaires et espèces comme précurseurs de particules secondaires) dans la région franco-belge, et l'occurrence de conditions météorologiques propices à la formation de particules secondaires, et donc la nécessité de mettre en place des études pour approfondir les connaissances sur ces processus de formation et identifier les leviers pour améliorer la qualité de l'air de la région. "

de nouvelles particules, sous l'effet de conditions météorologiques propices. Certains épisodes de pollution observés en Hauts-de-France sont d'ampleur nationale, c'est par exemple le cas de l'épisode de pollution à l'ozone du 22 au 27 juillet 2019 qui a touché la plupart des régions métropolitaines ${ }^{5}$, dont les Hauts-de-France du 23 au 26 juillet.

5. Bilan de la qualité de l'air extérieur en France en 2019. Ministère de la Transition écologique, septembre 2020, p. 19. 


\section{Les liens entre qualité de l'air et météorologie}

Les concentrations en polluants dans l'air dépendent de plusieurs facteurs : les quantités de polluants émises, leurs propriétés chimiques et physiques, et les conditions météorologiques. Ainsi, certaines situations météorologiques sont plus favorables que d'autres à l'accumulation des polluants et donc au déclenchement d'épisodes de pollution.

Le vent intervient sur la dispersion des polluants dans l'atmosphère : il brasse les polluants. Sa direction oriente les panaches de fumée par exemple, alors que sa vitesse aura des conséquences sur le déplacement des polluants. Plus le vent est fort (conditions cycloniques), plus il disperse les polluants. $\mathrm{Au}$ contraire, des situations anticycloniques persistantes (pression atmosphérique élevée et temps sec) ou de " marais barométriques » (peu de variation de pression sur de longues distances) peuvent aussi engendrer une accumulation progressive des polluants. Les vents peuvent transporter les polluants sur des distances plus ou moins grandes. Par exemple, l'ozone et les particules peuvent être transportés sur plusieurs centaines de kilomètres. Les mouvements convectifs favorisent la dispersion des polluants et les brises de mer peuvent amener soudainement de l'air peu pollué ou très pollué depuis la mer vers les zones côtières habitées.

Des températures élevées et un fort ensoleillement favorisent la formation de certains polluants dans l'atmosphère. Par exemple, l'ozone (Chanin et al., 2015) se forme grâce à un processus photochimique favorisé par la chaleur et l'ensoleillement à partir de gaz déjà présents dans l'atmosphère (figure 5) : le dioxygène, les composés organiques volatils et le dioxyde d'azote. Les épisodes de pollution à l'ozone sont donc plus fréquents au printemps et pendant l'été.

La pluie favorise en général une bonne qualité de l'air. Les précipitations " lessivent » l'atmosphère en entraînant au sol certains polluants (particules, polluants gazeux solubles, etc.). De plus, la pluie est souvent associée à une atmosphère instable (situation dépressionnaire) qui contribue également à la dispersion des polluants. Cependant, l'humidité, la brume et le brouillard peuvent favoriser la formation de particules et d'autres polluants.

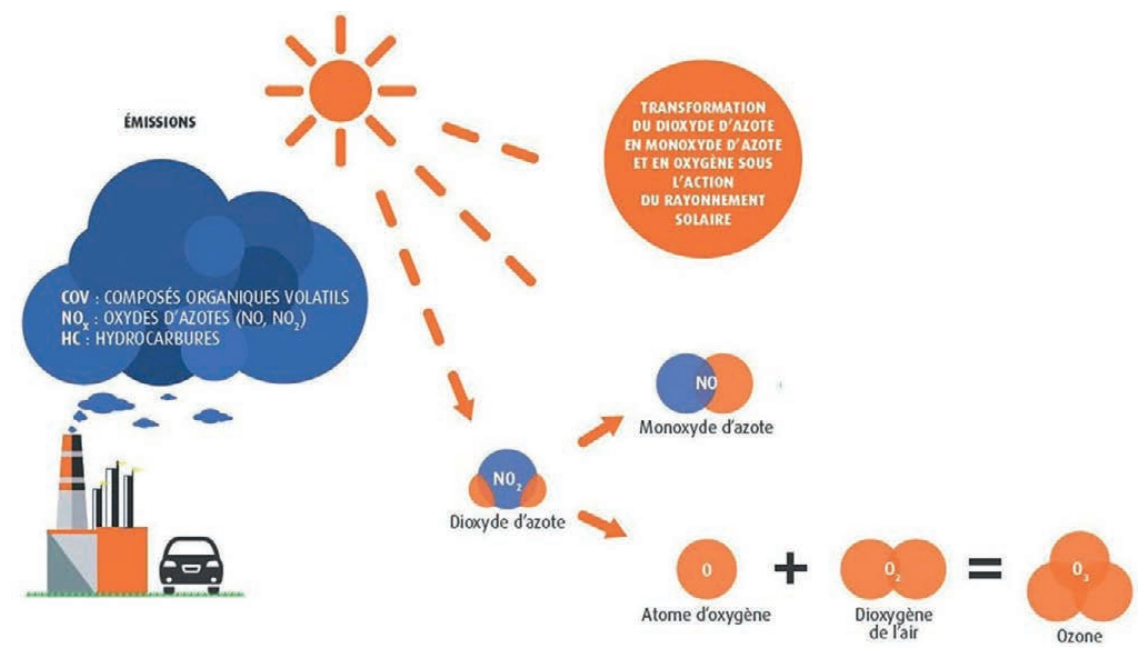

Figure 5. Représentation schématique de la formation de l'ozone. (c) Atmo Hauts-de-France.

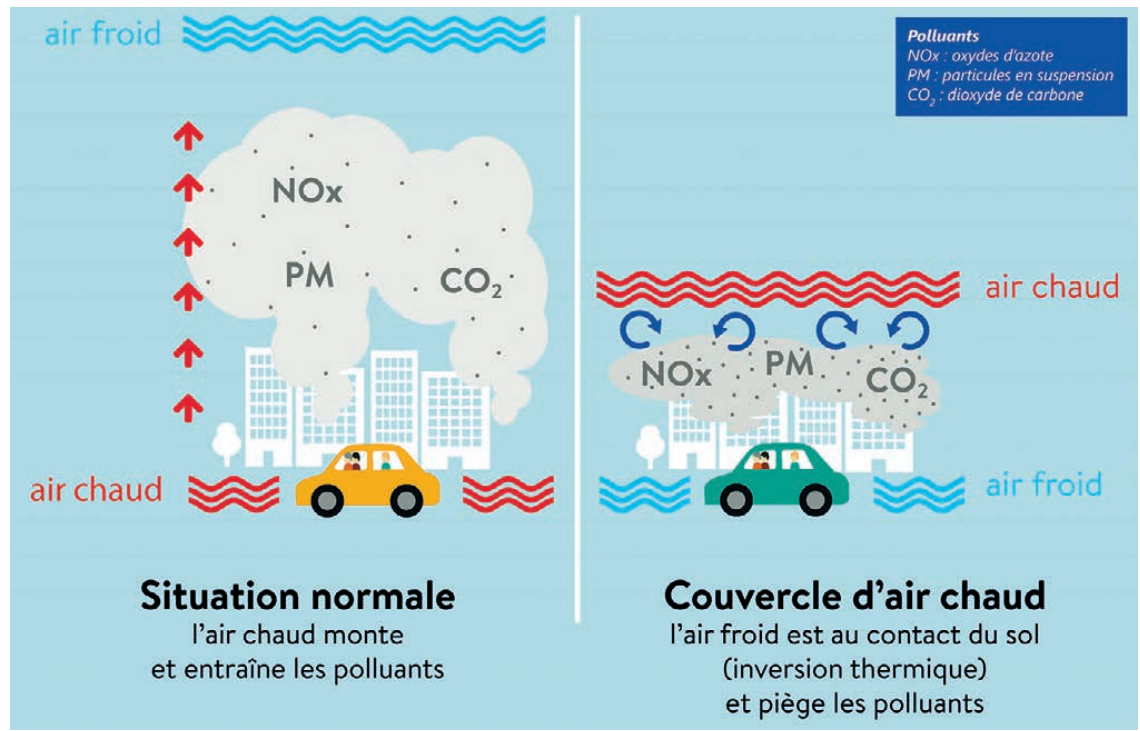

Figure 6. Représentation schématique d'une situation permettant la dispersion verticale des polluants (à gauche) et d'une situation d'inversion thermique qui bloque les polluants au sol (à droite). (c) Atmo Hauts-de-France.

La hauteur de la couche limite détermine la hauteur de l'atmosphère dans laquelle les polluants sont bien mélangés. Plus elle est grande, plus les polluants sont dilués verticalement. Elle dépend en particulier de l'ensoleillement ; ainsi, elle est en moyenne plus faible la nuit, augmente en journée et est maximale l'après-midi. En général, les conditions de dispersion sont donc meilleures en été qu'en hiver.

Les inversions de température favorisent l'accumulation des polluants au niveau du sol (figure 6). Dans la troposphère, la température de l'air diminue avec l'altitude (environ $0,65{ }^{\circ} \mathrm{C}$ tous les 100 mètres). L'air chaud s'élève dans les couches supérieures plus froides et entraîne avec lui les polluants qui sont ainsi dispersés de façon verticale. En hiver, la nuit fait chuter la température de l'air au niveau du sol et provoque la formation d'une " couche d'inversion ". L'air froid, ainsi que les brumes et les brouillards, se retrouvent bloqués sous cette couche d'inversion. Les polluants provenant des chauffages, des transports et des activités agricoles et industrielles s'accumulent sous cette couche d'air plus chaud, qui agit comme un couvercle. Le matin, sous l'effet du soleil, le sol se réchauffe, puis transfère sa chaleur à l'air situé près du sol. L'inversion de température s'élève alors et peut disparaître plus tard dans la journée si l'ensoleillement est suffisant.

D'autres phénomènes de formation d'inversion de température existent, par exemple lorsqu'une brise marine fraîche se glisse sous une couche d'air continental plus chaud. 
Certains paramètres météorologiques peuvent avoir des effets multiples sur les polluants. La topographie et l'occupation des sols (bâtis) peuvent accentuer ou atténuer l'effet des phénomènes météorologiques (turbulence des masses d'air, direction et vitesse des vents). La détermination de l'effet combiné de tous les paramètres météorologiques sur les concentrations en polluants est donc complexe et nécessite souvent l'analyse quotidienne d'un grand nombre de données par les prévisionnistes des Aasqa.

\section{Perspectives}

En France, les Aasqa sont agréées par le ministère en charge de l'Écologie pour surveiller la qualité de l'air de leur région. Le réseau des Aasqa œuvre quotidiennement à harmoniser un certain nombre de leurs données et leur communication à l'échelle nationale, tout en gardant leurs spécificités régionales. Pour mener à bien les missions qui lui sont confiées et répondre aux enjeux du territoire, Atmo Hauts-de-France s'appuie sur plus de 40 ans d'expertise, ainsi que sur des ressources et des outils en constante évolution pour devenir toujours plus précis, fiables et exhaustifs. Elle participe également à l'amélioration des connaissances de certains polluants, comme les particules qui représentent un enjeu régional pour la santé des populations et pour l'environnement. Engagé depuis plusieurs années, le programme «Particules » piloté par Atmo Hauts-de-France a permis d'apporter des éléments de réponse sur les origines et la composition des particules dans la région ${ }^{6}$. La poursuite de ces travaux sur les particules ultrafines, le carbone suie, la caractérisation physico-chimique des particules, l'ammoniac, les épisodes de pollution, etc. ont pour objectif de mieux comprendre leurs origines pour permettre d'identifier des leviers d'actions aux niveaux local et régional. De même, afin d'améliorer la prévision des épisodes de pollution, Atmo Hauts-de-France développe ses outils de modélisation fine échelle pour réaliser à terme de la prévision journalière et horaire sur l'ensemble de la région avec une résolution de 25 mètres. Cet outil permettra de mieux anticiper ces phénomènes et d'informer les autorités afin de protéger la santé des populations. De plus, l'observatoire régional travaille étroitement avec l'Agence régionale de santé sur des messages et des recommandations sanitaires en lien avec la qualité de l'air au quotidien pour les différents publics de la région. Enfin, Atmo Hauts-de-France s'attache à impliquer les acteurs du territoire (citoyens, collectivités, acteurs économiques, scolaires, etc.) dans la surveillance de la qualité de l'air. Les objectifs sont multiples : recueillir les attentes des territoires et des différents acteurs sur la thématique de l'air, proposer des pistes d'actions ou des dispositifs de sensibilisation pour une meilleure compréhension des enjeux liés à la qualité de l'air et favoriser les actions individuelles et collectives pour l'améliorer. Depuis quelques années, plusieurs projets de participation citoyenne ont amorcé cette dynamique en proposant, par exemple, le suivi de polluants atmosphériques grâce à des microcapteurs individuels. L'enjeu des prochaines années pour les Aasqa sera d'étudier la faisabilité et la pertinence d'intégrer ces moyens de surveillance dans leur dispositif réglementaire.

6. Atmo HdF, Synthèse du Programme Particules (2013-2019) en Hauts-de-France, 2020

\section{Pour en savoir plus :}

Site d'Atmo France : atmo-france.org

Site d'Atmo Hauts-de-France : www.atmo-hdf.fr

Site du LCSQA : www.lcsqa.org/fr

Plateforme ODO de déclaration des odeurs : www.atmo-odo.fr

Programme régional de surveillance de la qualité de l'air (PRSQA) des Hauts-de-France : www.atmo-hdf.fr/atmo/programme-de-surveillance.html

Plan national de surveillance de la qualité de l'air ambiant (PNSQA) : www.ecologie.gouv.fr/sites/default/files/03_PNSQA_VF.pdf

Plateforme Prev'Air : www2.prevair.org

Modélisation fine échelle journalière sur les Hauts-de-France : sig.atmo-hdf.fr/portal/ apps/MapSeries/index.html?appid=a89e88c0b2164892876ca61e5709b516

\section{Bibliographie}

Boy D., 2020. Les représentations sociales de l'effet de serre et du changement climatique. Ademe, Rapport RCB Conseil, 40 p.

Chanin M.-L., Clerbaux C., Godin-Beekmann S., 2015. L'évolution de l'ozone troposphérique. Le point en 2015. Rapport de l'Académie des sciences.

Davis D.L., 2002. A look back at the London smog of 1952 and the half century since. Environnemental Health Perspectives, $110,12$.

Desmettres P., 2013. Évaluation des pesticides dans les exploitations agricoles. Atmo HdF, août 2013.

Desmettres P., 2015. 10 ans de surveillance des pesticides dans I'air à Lille. Atmo HdF, septembre 2015.

Escat E., 2013. Étude sur les résidus de produits phytosanitaires dans l'air en Picardie. Atmo Picardie, février 2013.

Le Roux E., 2020. Comparaison des niveaux de PM10 et PM2.5 inter-AASQA en 2019. Atmo Hauts-de-France. https://www.atmo-hdf.fr/joomlatools-files/docman-files/ Rapport_et_synthese_etudes/2021/note_comparaison_PM interAASQA_2019.pdf

Quindroit P., 2020. Mesure des pesticides dans les Hauts-de-France en 2018-2019. Atmo HdF, juillet 2020.

Zimmer A., 2013. Le brouillard mortel de la vallée de la Meuse (décembre 1930). Naturalisation de la catastrophe. Histoire, 115-131. 